\title{
Total intravenous anesthesia with propofol and remifentanil in a patient with MELAS syndrome -A case report-
}

\author{
Jin Suk Park, Chong Wha Baek, Hyun Kang, Su Man Cha, Jung Won Park, Yong Hun Jung, \\ and Young-Cheol Woo
}

Department of Anesthesiology and Pain Medicine, College of Medicine, Chung-Ang University, Seoul, Korea

A 23-year-old woman with MELAS (mitochondrial myopathy, encephalopathy, lactic acidosis, and stroke-like episodes) underwent a laparoscopy-assisted appendectomy. MELAS syndrome is a multisystemic disease caused by mitochondrial dysfunction. General anesthesia has several potential hazards to patients with MELAS syndrome, such as malignant hyperthermia, hypothermia, and metabolic acidosis. In this case, anesthesia was performed with propofol, remifentanil TCI, and atracurium without any surgical or anesthetic complications. We discuss the anesthetic effects of MELAS syndrome. (Korean J Anesthesiol 2010; 58: 409-412)

Key Words: Atracurium, General anesthesia, MELAS syndrome, Propofol, Remifentanil, TIVA.

MELAS (mitochondrial myopathy, encephalopathy, lactic acidosis, and stroke-like episodes) syndrome is caused by a mitochondrial dysfunction that affects many organs; mutations in the genes in the mitochondrial DNA, which is inherited from the female parent, cause MELAS [1]. The mitochondrion produces ATP through the Krebs cycle and the electro-transfer system. It also plays a role in apoptosis-programmed cell death, cell division, steroid synthesis, regulation of cellular metabolism, and heat production in tissues. The mitochrondion is able to replicate and divide according to the cell's energy needs. Diseases caused by mitochondrial dysfunctions, such as MELAS syndrome, have clinical manifestations predominantly in organs that require a lot of energy, such as brain, lungs, liver, and kidneys. Patients with the MELAS syndrome have reportedly experienced malignant hyperthermia, hypothermia, and resistance to muscle relaxants or their prolonged effects [2-6]. This case report describes the experiences with total intravenous anesthesia using target-controlled infusion of propofol and remifentanil to a MELAS syndrome patient undergoing a laparoscopic appendectomy.

\section{Case Report}

A 23 year-old female patient, $160 \mathrm{~cm}$ tall and $48 \mathrm{~kg}$, was diagnosed with acute appendicitis and admitted for a laparoscopic appendectomy. She had no abnormalities in her

Received: May 27, 2009. Revised: 1st, June 15, 2009; 2nd, July 1, 2009. Accepted: July 7, 2009.

Corresponding author: Chong Wha Baek, M.D., Department of Anesthesiology and Pain Medicine, College of Medicine, Chung-Ang University, 224-1, Heukseok-dong, Dongjak-gu, Seoul 156-070, Korea. Tel: 82-2-6299-2583, Fax: 82-2-6299-2585, E-mail: nbjhwa@naver.com (c) This is an open-access article distributed under the terms of the Creative Commons Attribution Non-Commercial License (http:// creativecommons.org/licenses/by-nc/3.0/), which permits unrestricted non-commercial use, distribution, and reproduction in any medium, provided the original work is properly cited. 
pediatric medical history, but from the age 18, she had slowly begun experiencing headaches in her left temporal lobe, nausea, and vomiting. In June of that year, she experienced generalized convulsions and aphasia. In a brain MRI and MRA, an infarction in the posterior divisions of the left mesencephalic arteries was found. Also, in a blood test, an increase in lactic acid was discovered (7.54 mM/L [normal level: 0.5-2.254 mM/ L]). The patient was suspected to have MELAS syndrome. She was diagnosed with MELAS after testing (adenosine-to-guanine transition at t-RNA nucleotide 3243 in PCR sequencing). After she turned 21, she was diagnosed with Type I DM. The patient had to orally ingest $200 \mathrm{mg}$ of carbamazepine and $100 \mathrm{mg}$ of aspirin per day and subcutaneously inject 30 units of insulin in the morning and 20 units in the evening. She had no history of total anesthesia.

In a physical examination before the anesthesia, the patient was able to read but had auditory aphasia such that she could not understand spoken words. In the physical examination, there were no signs of hypotonia or amyotrophy of the limbs. The laboratory results showed hyponatremia (126 mEq/L), hyperglycemia $(257 \mathrm{mg} / \mathrm{dl})$, and light metabolic acidosis $(\mathrm{pH}$ 7.346, $\mathrm{PaCO}_{2} 36.3 \mathrm{mmHg}, \mathrm{HCO}_{3} 20.1 \mathrm{mM} / \mathrm{L}, \mathrm{BE}-5.3 \mathrm{mM} /$ $\mathrm{L})$. There were no abnormal findings in the chest $\mathrm{X}$-ray and electrocardiogram. One year prior to her admission to the hospital, she had an ECG that revealed a cardiac index of $67 \%$ and no abnormal findings. For sugar control, 4 units of shortacting insulin were injected, and $0.9 \%$ normal saline was administered to control hyponatremia. No other pre-operative measures were performed.

After the patient was taken to the operating room, we attached ECG standard leads II, noninvasive monitors for blood pressure, heart rate, arterial oxygen saturation, capnogram, and bispectral index (BIS), and a nerve stimulator to the patient using the Multi Channel Anesthesia Monitor S/5 ${ }^{\mathrm{TM}}$ (Datex-Ohmeda, USA). Preliminary vital signs were as follows: blood pressure 115/65 $\mathrm{mmHg}$, heart rate 100 beats/min, oxygen saturation rate $97 \%$, and the ECG results appeared normal. The patient underwent 3 minutes of denitrogenation with $100 \%$ oxygen through a face mask. Afterwards, we administered lidocaine (40 mg) with the Master TCI (Fresenius Vial S.A., France); we then injected 2\% propofol (Fresofol ${ }^{\circledR}$, Fresenius Kabi, Austria) and remifentanil (Ultiva $^{\mathrm{TM}}$, GlaxoSmithKline, UK) at target concentrations of 4 $\mu \mathrm{g} / \mathrm{ml}$ (Marsh-model) and $5 \mathrm{ng} / \mathrm{ml}$ (Minto-model), respectively. After roughly 90 seconds had passed, we checked lid reflexes and found that the patient had lost consciousness. We administered atracurium $(0.5 \mathrm{mg} / \mathrm{kg})$, the BIS was 50 , and we saw that there was no response to TOF stimulation with the nerve stimulator placed on the ulnar nerve. Endotracheal intubation was then performed without complications. We started mechanical respiration with air (1.5 L/min), oxygen (1.5
$\mathrm{L} / \mathrm{min}$ ), respiratory volume of $450 \mathrm{ml}$, and respiratory rate of 12 breaths per minute. To control ventilation, the capnogram was kept at 35-40 mmHg. We used spirometry to measure the respiratory volume and pulmonary compliance. We started invasive blood pressure monitoring through the radial artery and measured the esophageal temperature with a body temperature monitor. During the operation, we used a forced-air warming blanket (Bair Hugger ${ }^{\mathrm{TM}}$, Austine Medical, USA) to stabilize the patient's body temperature. For fluid maintenance, we administered $0.9 \%$ normal saline at $200 \mathrm{ml}$ per hour. During the operation, the patient's vitals were kept stable with blood pressure at $120-140 / 60-80 \mathrm{mmHg}$, heart rate $80-100$ beats/ min, oxygen saturation $100 \%$, body temperature $37.0-37.4^{\circ} \mathrm{C}$, and BIS 40-60. Thirty minutes after inducing anesthesia, the arterial blood gas study showed $\mathrm{pH} 7.44, \mathrm{PaCO}_{2} 32 \mathrm{mmHg}$, $\mathrm{PaO}_{2} 299 \mathrm{mmHg}, \mathrm{HCO}_{3} 23.3 \mathrm{mM} / \mathrm{L}, \mathrm{BE}-2.1 \mathrm{mM} / \mathrm{L}$; her electrolytes were $\mathrm{Na}^{+} 125 \mathrm{mEq} / \mathrm{L}, \mathrm{K}^{+} 3.8 \mathrm{mEq} / \mathrm{L}, \mathrm{Cl}^{-} 88 \mathrm{mEq} /$ $\mathrm{L}$; and her blood sugar was $176 \mathrm{mg} / \mathrm{dl}$. Twenty minutes prior to the end of the operation, we administered ondansetron (4 mg) to prevent post-operative nausea and vomiting. After suturing the peritoneum, we stopped injecting remifentanil, kept the level of propofol in the target effect site at $2 \mu \mathrm{g} / \mathrm{ml}$, and restored spontaneous breathing. After the operation was over, we stopped injecting propofol and stabilized spontaneous breathing at $300 \mathrm{ml}$ per breath on the spirometer. On the nerve stimulator, the TOF rate was kept at 0.95 . To reverse the muscle relaxant effects, we administered glycopyrrolate $(0.4 \mathrm{mg})$ and pyridostigmine (15 mg). Afterwards, the patient responded to voice commands and opened her eyes. With stabilized spontaneous breathing, she was extubated. The operation lasted around 1 hour, and during the operation, $350 \mathrm{ml}$ of $0.9 \%$ normal saline were used. We then moved the patient to the recovery room and kept her under observation with blood pressure, electrogram, and oxygen saturation monitors. In the recovery room, we gave her oxygen at $5 \mathrm{~L} / \mathrm{min}$ through the facial mask. The arterial blood gas study showed $\mathrm{pH} 7.32, \mathrm{PaCO}_{2} 43 \mathrm{mmHg}$, $\mathrm{PaO}_{2} 219 \mathrm{mmHg}, \mathrm{HCO}_{3} 21.2 \mathrm{mM} / \mathrm{L}$, and $\mathrm{BE}-3.6 \mathrm{mM} / \mathrm{L}$; her electrolyte levels were $\mathrm{Na}^{+} 128 \mathrm{mEq} / \mathrm{L}, \mathrm{K}^{+} 4.1 \mathrm{mEq} / \mathrm{L}$, and $\mathrm{Cl}^{-}$ $90 \mathrm{mEq} / \mathrm{L}$; and her blood sugar level was $120 \mathrm{mg} / \mathrm{dl}$. We then moved the patient to the ward where we performed a lactic acid test, which measured $3.6 \mathrm{mM} / \mathrm{L}$. Three days after the operation, the patient showed no complications from the operation or anesthesia, so she was released from the hospital.

\section{Discussion}

The mitochondrion synthesizes ATP from ADP and inorganic phosphate through the respiration chain. Thus, mitochondrial diseases can cause all kinds of organ dysfunctions. Clinical manifestations have been found in tissues with high levels of 
energy consumption, such as brain and muscles. Kearns-Sayre syndrome, MELAS syndrome, mitochondrial myopathy, DAD (Diabetes mellitus and deafness), LHON (Leber's hereditary optic neuropathy), Leigh syndrome, NARP (neuropathy, ataxia, retinitis pigmentosa, and ptosis), MERRF (myoclonic epilepsy with ragged red fibers) syndrome, and MNGIE (Myoneurogenic gastrointestinal encephalopathy) are all categorized as mitochondrial diseases.

MELAS syndrome was first described by Pavlakis in 1984. It is characterized by dementia and seizures caused by encephalopathy, lactic acidosis, ragged-red fibers, and strokelike episodes that usually occur before the age of 40 . There is also a build-up of lactic acid in plasma and cerebrospinal fluid. This mitochondrial disease is inherited from the female parent and is caused by an A-to-G transition mutation at position 3243 of the mitochondrial genome. There is currently no curative treatment for the disease, but there have been various attempts of symptomatic treatment [1].

Because MELAS syndrome causes systemic diseases that affect many organs, including the brain, a patient who needs to undergo general anesthesia will require careful anesthetic monitoring, especially of body temperature. Fricker et al. [2] reported a patient with mitochondrial myopathy accompanied by myoadenylate deaminase deficiency who was highly sensitive to his malignant hyperthermia. However, Morgan et al. [7] and Burns et al. [8] reported that there was no significant change in the patient's body temperature when using sevoflurane and isoflurane for general anesthesia. There are controversies about the relationship between MELAS syndrome and malignant hyperthermia, but because MELAS syndrome is a mitochondrial disease, we must beware of malignant hyperthermia. Also during an operation, we must be careful of hypothermia because it can further inhibit mitochondrial functions [3]. In our case, we performed total intravenous anesthesia using propofol and remifentanil to prevent malignant hyperthermia; to prevent hypothermia, we continuously monitored body temperature via thermometer placed in the esophagus and used a forced-air warming blanket.

When performing total intravenous anesthesia, propofol must be used with care. Propofol can cause mitochrondial dysfunction, which is thought to be caused by the propofol infusion syndrome from extended use of propofol [9]. Cautionary use of propofol is therefore suggested in long operations. The present case was a short, 1-hour operation, so we did not experience any complications such as propofol-induced changes in heart functions or worsening of acidemia midoperation.

MELAS patients have varied experiences with non-depolarizing muscle relaxants. Finsterer et al. [4] and Wisely et al. [5] reported on mitochondrial myopathy patients who suffered prolonged effects from rocuronium and vecuronium. Aouad et al. [6] reported on MELAS patients with resistance to cisatracurium. Tempelhoff et al. [10] also reported patients resistant to nondepolarizing muscle relaxants like the patient in our case; they stated that patients on anticonvulsants like carbamazepine and phenytoin experienced resistance. Because many MELAS patients also contract kidney disorders, for example, de ToniDebre-Fanconi syndrome, nephritic range proteinuria, and FSGS [1], causing a disorder in the excretion of drugs or extending the effects of the medicine, one should refrain from the usage of nephrotoxic drugs and drugs excreted by the kidneys. Thus, for our patient, we used atracurium, which is not excreted by the kidneys. We continuously checked the level of muscle relaxation and found neither prolonged effects nor resistance to the muscle relaxant.

MELAS patients need to be carefully monitored for acidemia and electrolyte imbalance and require fluid therapy accordingly. Most patients have lactic acidosis and metabolic acidosis. Administering a fluid containing lactic acid, such as Ringer's lactate solution, will worsen lactic acidosis, which will also cause metabolic acidosis. Because our patient had hyponatremia and lactic acidosis, we injected $0.9 \%$ normal saline. The patient's hyponatremia was not corrected after the operation, but because she did not appear to have hyponatremia-caused reduced level of consciousness after regaining consciousness, it was not addressed.

Problems in the cardiovascular system including cardiomyopathy, WPW syndrome, and conduction disturbances are prevalent in MELAS patients [1]. Therefore, having an ECG prior to an operation is absolutely necessary, Echocardiography tests should be taken if possible, and continuous ECG monitoring and blood pressure monitoring are necessary during the operation and immediately thereafter. Moreover, the usage of drugs that cause changes in the cardiovascular system should be avoided or used carefully in small amounts during the operation.

Diabetes is the most prevalent disease in MELAS patients [1]. When the patient has to fast for the surgery, the tissue cells cannot get enough energy for fast metabolism, and build-up of metabolites such as lactic acid worsens the lactic acidosis. Therefore, before and after surgery, the patient's blood sugar level must be carefully controlled with particular caution so that the patient does not experience hypoglycemia during anesthesia. In the present case, we administered short-acting insulin to the patient for hyperglycemia. Also, before, during, and after anesthesia in the recovery room, we regularly monitored her blood sugar level, but the patient did not have significant hypoglycemia or hyperglycemia.

In conclusion, because MELAS syndrome affects all organs, particularly in the excretory system, cardiovascular system, 
and respiratory system, general anesthesia for MELAS patients requires special attention to the choices of the anesthetic method, drugs, and fluid. Therefore, in pre-anesthetic evaluations, one must take all symptoms of the body into consideration and carefully monitor the patient for various complications, such as malignant hyperthermia, hypothermia, prolongation of muscle relaxant effects, and instability in the cardiovascular system, during the operation.

\section{References}

1. Sproule DM, Kaufmann P. Mitochondrial encephalopathy, lactic acidosis, and strokelike episodes. basic concepts, clinical phenotype, and therapeutic management of MELAS syndrome. Ann NY Acad Sci 2008; 1142: 133-58.

2. Fricker RM, Raffelsberger T, Rauch-Shorny S, Finsterer J, MüllerReible C, Gilly H, et al. Positive malignant hyperthermia susceptibility in vitro test in a patient with mitochondrial myopathy and myoadenylate deaminase deficiency. Anesthesiology 2002; 97: 1635-7.
3. Cholley F, Edery P, Ricquier D, Peudenier S, Slama A, Tardieu M. Mitochondrial respiratory chain deficiency revealed by hypothermia. Neuropediatrics 2001; 32: 104-6.

4. Finsterer J, Stratil U, Bittner R, Sporn P. Increased sensitivity to rocuronium in mitochondrial myopathy. Can J Anaesth 1998; 45: 781-4.

5. Wisely NA, Cook PR. General anesthesia in a man with mitochondrial myopathy undergoing eye surgery. Eur J Anaesthesiol 2001; 18: 333-5.

6. Aouad MT, Gerges FJ, Bararka AS. Resistance to cisatracurium in a patient with MELAS syndrome. Paediatr Anaesth 2005; 15: 1124-7.

7. Morgan PG, Hoppel CL, Sedensky MM. Mitochondrial defects and anesthetic sensitivity. Anesthesiology 2002; 96: 1268-70.

8. Burns AM, Shelly MP. Anaesthesia for patients with mitochondrial myopathy. Anaesthesia 1989; 44: 975-7.

9. Vasile B, Rasulo F, Candiani A, Latronico N. The pathophysiology of propofol infusion syndrome: a simple name for a complex syndrome. Intensive Care Med 2003; 29: 1417-25.

10. Tempelhoff R, Modica PA, Jellish WS, Spitznagel EL. Resistance to atracurium-induced neuromuscular blockade in patients with intractable seizure disorders treated with anticonvulsants. Anesth Analg 1990; 71: 665-9. 